\title{
Automatic obstacle avoidance and parietal cortex
}

\author{
Glyn W Humphreys \& Martin G Edwards
}

\begin{abstract}
The ventral visual stream is proposed to support perception, and the dorsal stream is proposed to control action. A new study of patients with posterior parietal damage shows that the dorsal stream also contributes to automatic obstacle avoidance in reaching.
\end{abstract}

People reaching for a drink across a crowded bar avoid the obstacles in their way without thinking about it. Yet obstacle avoidance has proven surprisingly difficult to achieve in robotic applications, where movements must be made in cluttered and sometimes dangerous environments. In this issue, Schindler and colleagues ${ }^{1}$ report that automatic obstacle avoidance in visually guided reaching is supported by the dorsal visual stream. People reaching between two objects have a strong tendency to alter their reach trajectory in response to changes in obstacle position, even if the objects are far enough away that they do not interfere with the arm's path. In contrast, the new work shows that people with damage to the posterior parietal lobe fail to adjust their reaching responses according to the locations of such irrelevant objects. The authors conclude that the dorsal stream provides a kind of automatic visual guidance during reaching behavior, tuned not only to the location of a target object but also to avoiding irrelevant objects in the scene.

Schindler et al. ${ }^{1}$ studied reaching in two individuals with optic ataxia-a disorder found after posterior parietal damage and associated with impairment of visually guided actions ${ }^{2}$. Subjects were asked to reach between two cylinders to touch a central target strip (the reach task) or to point to the center of the space between the two cylinders (the bisection task). Normal participants in the reach task systematically shifted their responses according to the position of the cylinders, even though they were not instructed to do so. For example, when the cylinders were shifted to the right, normal reaches between them passed farther to the right. Strikingly, the subjects with optic ataxia did not adjust the path of their reaches as the positions of the irrelevant cylinders varied. In contrast, the ataxic subjects performed like normal controls in the bisection

Glyn W. Humphreys is in the Behavioural Brain Sciences Centre, School of Psychology, and Martin G. Edwards is in the School of Sport and Exercise Sciences, University of Birmingham, Birmingham, B15 2TT, UK

e-mail: g.w.humphreys@bham.ac.uk task, when they were instructed to locate the midpoint between the obstacles, adjusting their pointing response as the locations of the cylinders were varied. Thus the ataxic subjects could take account of the obstacles' positions when they were asked to consciously estimate the midpoint, but the same stimuli did not influence a more automatic reach action as it does in normal controls.

These new data ${ }^{1}$ also contrast with results from people with other neuropsychological disorders, such as visual agnosia, involving damage to the ventral visual stream. Agnosic individuals can take account of obstacles when reaching but have problems pointing to the midpoint between obstacles (R.D. McIntosh, H.C. Dijkerman, M. Mon-Williams \& A.D. Milner, presentation at the Experimental Psychology Society, Cambridge, 2000)—a pattern opposite to that seen in optic ataxia. Schindler et al. take this double dissociation as indicative of the distinctive roles of the ventral and dorsal streams in vision. A current view is that the ventral stream supports perceptual judgments (such as judgments about the midpoint between two stimuli) whereas the dorsal stream mediates the on-line control of action $^{3}$. From their results, Schindler et al. propose that the dorsal stream also computes reach trajectories that avoid potential obstacles. As a consequence, when this processing stream is damaged, obstacles no longer influence reaching performance.

Another interesting aspect of the new paper $^{1}$ is that, although the ataxic subjects planned their movements visually, the experimenters obscured the subjects' vision when they started to move. This, in turn, suggests that the dorsal stream is not only involved in the on-line control of action but also takes account of obstacles in the environment when the movement is programmed, before the action occurs. Little is known about the role of the dorsal stream in programming action, and this is clearly an important area for future research. Also, as Schindler and colleagues note, little is known about which properties of the obstacles are important-do only location and size matter? Do other properties influence movement programming in the dorsal stream, or must other brain regions then be recruited?
Various alternative interpretations can be offered for these results, but the authors argue that these explanations are unlikely. For example, it might be that, relative to controls, the ataxic subjects take less account of the cylinders in reaching because of the difficulty they have in making arm movements, which are often slow and might require high levels of concentration. Obstacles may have less influence on slow movements, which are easier to control, and thus correct, on-line. However, analysis of the kinematics of the ataxic subjects' reaches showed that only one of the two tested had abnormally slow reaches. Thus the speed of the movement does not seem to be crucial. Also, when concentrating on the movement, ataxic individuals may pay less attention to irrelevant stimuli-as proposed by current theories of human attention ${ }^{4}$. However, Schindler et al. note that a subject with optic ataxia of the right limb from left posterior parietal damage failed to take into account only those obstacles on his right (contralesional) side; his performance was influenced normally by the position of obstacles on the relatively unaffected left (ipsilesional) side. Thus this individual did not show a generalized lack of attention resulting from concentration on the action.

Before discounting any role for attention, however, it may be important to examine how the influence of obstacles varies as a function of an person's current attentional load. Even someone with unilateral damage may show differential weighting of attention to the ipsilesional side, where obstacles are taken into account, and away from the contralesional side, where obstacles have reduced influence. Even if the dorsal stream is important for automatic aspects of action, this does not mean that attention does not modulate how it operates. Given other evidence for effects of attention on action ${ }^{5}$, we strongly suspect that such modulating effects could be present.

1. Schindler, I. et al. Nat. Neurosci. 7, 779-784 (2004). 2. Perenin, M.-T. \& Vighetto, A. Brain 111, 643-674 (1988).

3. Milner, A.D. \& Goodale, M.A. The Visual Brain in Action (Academic, London, 1995).

4. Lavie, N. J. Exp. Psych. Hum. Percept. Perform. 21, 451-468 (1995).

5. Humphreys, G.W et al. in Attention in Action (eds. G.W. Humphreys \& M.J. Riddoch) (Psychology Press, London, in press). 